\title{
El documental televisivo: un género en peligro de extinción en Europa
}

\author{
Gina PlanA \\ Universitat Autònoma de Barcelona \\ gina.plana@uab.cat \\ Emili PRADO \\ Universitat Autònoma de Barcelona \\ emili.prado@uab.cat
}

Recibido: 6 de noviembre de 2013

Aceptado: 19 de mayo de 2014

\section{Resumen}

En los años cincuenta parecía obvio que el hábitat natural del documental era la televisión y la evolución del género y la del medio fueron de la mano durante décadas. Desde entonces, no obstante, la imposición de los imperativos comerciales sobre la televisión ha comportado la modificación de las cualidades originales del género y ha supuesto la progresiva desaparición de los documentales de las parrillas programáticas. Este artículo analiza el estado actual de la cuestión, cuantificando y analizando los documentales aparecidos en el prime-time de las principales televisiones europeas. Los resultados demuestran una presencia mínima del documental en los mercados televisivos más importantes de Europa, exceptuando el Reino Unido.

Palabras clave: documental, Europa, reportaje, prime-time, televisión

\section{Television Documentary: an Endangered Genre in Europe}

\begin{abstract}
In the fifties it seemed obvious that the natural habitat of documentary films was television, and the genre and the medium evolved simultaneously for decades. Since then, however, the imposition of commercial imperatives on television has led to the modification of the original qualities of the genre and has meant the gradual disappearance of documentary films form the program grids. This article analyses the current state of the issue, quantifying and analysing the documentaries shown during the prime-time slot in the 5 major European television markets. The results demonstrate a minimum presence of documentaries in Europe, with the sole exception of the United Kingdom.
\end{abstract}

Keywords: documentary, Europe, report, prime-time, television

\section{Referencia normalizada}

PLANA, Gina y PRADO, Emili (2014): "El documental televisivo: un género en peligro de extinción en Europa". Estudios sobre el Mensaje Periodístico. Vol. 20, Núm. 2 (julio-diciembre), págs.: 841-856. Madrid, Servicio de Publicaciones de la Universidad Complutense.

Sumario: 1. Introducción. 2. Fuentes y Metodología. 3. La Presencia de documentales en el prime-time europeo; 3.1. Resultados por país (2010-2011); 3.1.1. Alemania; 3.1.2. España; 3.1.3. Francia; 3.1.4. Italia; 3.1.5. Reino Unido; 3.2. Resultados comparativos (UE-5 2010-2011). 4. Conclusiones. 5. Referencias bibliográficas.

1 Esta investigación se ha realizado en el marco del proyecto SEJ2009-18822 financiado por el MICINN, desarrollada en el Grupo de investigación GRISS de la Universitat Autònoma de Barcelona, reconocido y financiado por la Generalitat de Catalunya (2009SGR-1013) 


\section{Introducción}

En los años cincuenta parecía una obviedad que el hábitat natural del documental era la televisión; teóricos y profesionales coincidían en vaticinar un futuro de estrecha relación entre el género y el medio que debían aprovecharse el uno del otro a modo de simbiosis: si el documental obtenía de la televisión un medio privilegiado de difusión, el nuevo medio veía en el documental un formato idóneo para la información en profundidad, esencial para su cometido de servicio público. Era la Europa de postguerra, donde una televisión en plena efervescencia empezaba a desbancar el cine documental de las salas de exhibición cinematográfica (Francés, 2003: 133) y delineaba las bases del consumo audiovisual por venir.

Los años sesenta se caracterizaron por la aparición de las primeras televisiones privadas y la resultante mercantilización de la televisión trajo consigo algunas consecuencias con respecto al documental. En Inglaterra, por ejemplo, el nacimiento de la ITN supuso la creación de la BBC2 y el traslado de buena parte de los contenidos culturales a la segunda cadena, potenciando el entretenimiento y las noticias en la primera, con el fin de competir en audiencia con la televisión independiente. A diferencia de los Estados Unidos, donde la televisión nació ligada a la iniciativa privada, en Europa, la evolución del servicio público llevó consigo el mantenimiento del documental en las parrillas a lo largo de las décadas siguientes. Aquella televisión tenía el objetivo de "responder a las necesidades informativas, educativas y de diversión de los ciudadanos, además de promover la cultura nacional y el conjunto de valores de la civilización" (Richeri, 1994: 46-47). Se implantó un modelo de programación fundamentalmente vertical, al margen todavía de las influencias que recibiría más adelante de la televisión comercial, y se establecieron espacios para el documental en la mayoría de televisiones públicas de Europa (Francés, 2003: 138).

La des-regulación del modelo estatal en los setenta supuso una renovada tendencia hacia el modelo comercial de televisión caracterizado, según Bustamante, por "una serialización cada vez más sistemática [...], una más rápida frecuencia de emisión de los distintos productos, [...] una lógica horizontal que gana terreno progresivamente a la antigua lógica vertical, [...] y una tendencia al alargamiento de los bloques" (Bustamante, 1999: 99-100), aunque la mayoría de países europeos contaron con televisiones públicas hegemónicas, cuando no monopolísticas, hasta los ochenta. "El establecimiento de los sistemas de titularidad mixta pública/privada promueve un escenario competitivo que diluye la propia idea de servicio público [...] En ese contexto las cadenas públicas [...] han ido abandonando los tres ejes sobre los que teóricamente se articulaba su actuación en el pasado: informar, formar y entretener" (Prado, 1995: 86). Con la irrupción de las cadenas privadas la televisión pública se separa definitivamente de sus objetivos fundacionales en la mayoría de mercados, asumiendo el entretenimiento como contenido imprescindible, lo que desplaza el documental de las primeras cadenas.

Desde los 90 la tendencia ha sido unívoca en los grandes mercados europeos: los programas documentales se han vuelto más "blandos", han introducido elementos de ficción, se han serializado (Kilborn, 1998: 203; Zoellner, 2009: 504) y se han ido transformando; han surgido nuevos géneros híbridos como el docu-soap o docuglitz 
(Brundson et al., 2001: 31; Coles, 2000: 27; Kilborn, 2000: 111), han desaparecido de la franja de prime-time los documentales tradicionales (Prado, 2002: 387) y "sólo los segundos canales de las televisiones públicas han dejado posibilidad a la emisión de estos programas" (Pérez Ornia, 2000 en Francés, 2003: 143).

Hoy en día, parece que el género es poco o nada rentable en términos de audiencia y es por ello que las televisiones, tanto públicas como privadas, han optado por programarlo cada vez menos. "Documentaries are not as cost-effective in production as long-running series or show-formats that can be cheaply reproduced for other series" (Zoellner, 2010: 509). El punto de vista de las televisiones privadas es muy claro: el documental genera índices muy bajos y estos no cumplen las expectativas de los clientes publicitarios. Lo manifiestan las palabras de Dawn Airey, director de ITV, reproducidas por Anna Zoellner en "Professional Ideology and Program Conventions: Documentary Development in Independent British Television": "[ITV plc] is a business. To be frank something like a single documentary made for another broadcaster - there is no value in it beyond the production fee. I am not interested in running a 7\% production margin" (Thomas, 2008 en Zoellner, 2009: 504).

En el ámbito de la televisión pública podría presuponerse un panorama diferente, si se tienen en cuenta los objetivos fundacionales del medio así como el potencial del documental para cumplirlos. En principio, el documental puede informar, educar y entretener, es decir, asumir los objetivos fundamentales del espacio televisivo. (León, 2009: 15). En los últimos años, no obstante, el triple motivo acuñado por el histórico director de la BBC John Reith (Manfredi Sánchez, 2008: 29) ha dejado de ser una aspiración para convertirse en un modelo ideal que ya pocos intentan alcanzar, en un panorama globalmente dominado por el entretenimiento. Lord Reith expuso que el entretenimiento debía ser el último de los tres objetivos a cumplir por los medio públicos ya que la información y la educación eran los "objetivos nucleares para la difusión pública" (León, 2009: 15). Pero lo cierto es que estas cadenas, inmiscuidas en la misma competición por la audiencia que los operadores privados, a menudo utilizan iguales criterios de programación que la televisión comercial. En el caso de Inglaterra, Zoellner cita las palabras del comissioning editor de la BBC quien, al ser preguntado por las tres cosas que quería que proporcionara una buena película respondió simplemente "ratings, ratings and ratings" (Zoellner, 2009: 521).

Esta tendencia lleva consigo la reducción de las posibilidades del género a sus opciones comerciales. En Europa, y en el mundo entero, el documental intenta sobrevivir en un panorama televisivo dominado por la economía, lo que resulta en la creación de nuevos géneros de actualidad, más accesibles y más blandos. (Chapman y Allison, 2009: 9). Estos datos, probados hasta el momento, se actualizan y matizan con los resultados de esta investigación, que tiene por objeto diagnosticar la salud de los documentales en televisión, en una época en la que el liderazgo en los macrogéneros se ha otorgado al entretenimiento en todos los mercados europeos (Prado y Delgado, 2010: 54). El objetivo principal es dibujar un panorama a través de la observación sistemática y el análisis científico de la programación de prime-time, en base a los objetivos siguientes: 
- Cuantificar la presencia de documentales y grandes reportajes en el prime-time de las principales televisiones de cinco países europeos: España, Reino Unido, Francia, Italia y Alemania.

- Establecer en qué países y en qué televisiones se programan más documentales en horario de prime-time.

- Clasificar los documentales emitidos en el prime-time en términos de formato y contenido.

- En las televisiones públicas, establecer la relación entre la cantidad de documentales programados y las distintas formas de financiación de las cadenas.

\section{Fuentes y metodología}

A la hora de plantearse la metodología necesaria para la investigación hace falta tener en cuenta que el objetivo principal se resuelve en el dibujo de un panorama y en la interpretación del mismo. Por ello, la técnica cuantitativa imprescindible es el análisis de contenido, ya que permite una descripción objetiva, sistemática y cuantitativa del contenido manifiesto mediante la cuantificación de las variables (Berelson,1952: 17). Esta técnica se ha aplicado en tres sentidos pero en un mismo momento:

a) Recuento de los documentales en el horario de prime-time de las principales televisiones escogidas.

b) Tipificación y clasificación de los documentales aparecidos en base a los parámetros descritos en la Tabla 1. En esta fase, el análisis de contenido permite caracterizar a un nivel básico todos los documentales con presencia en el primetime.

c) Interpretación de los resultados, asumiendo criterios valorativos.

El desarrollo metodológico ha consistido en registrar y visionar todos los contenidos comprendidos en el prime-time de los países seleccionados: Alemania España, Francia, Italia y Reino Unido. Después, se han identificado los programas documentales, para los cuales se han elaborado fichas de análisis en forma de tabla con 15 variables. Las variables contempladas para la caracterización de cada documental son las siguientes:

\begin{tabular}{lll} 
& \multicolumn{2}{c}{ Tabla1. Variables } \\
\hline - País & - Título & \\
- Canal & - Hora de inicio & - Espacio fijo o no \\
- Titularidad & - Hora finalización & - Dirección \\
- Fecha & - Duración & - Producción \\
- Día & - Año producción & - Formato \\
\hline
\end{tabular}

Fuente : Elaboración Propia

Elaboradas las tablas, el proceso ha finalizado con la visualización sistemática de los contenidos, acompañada del relleno de las tablas. La última tarea ha consistido en cruzar los datos con el fin de obtener los resultados requeridos y la valoración de los 
mismos. El análisis cruzado de las diversas variables dibuja el panorama perseguido como objeto de estudio.

Por su notoriedad académica y por su accesibilidad, se toman como parámetros de delimitación de la muestra los utilizados por los investigadores del Observatorio Euromonitor $^{2}$, analizando la programación de las 26 cadenas hertzianas de mayor audiencia de los cinco mercados televisivos más importantes de Europa, esto es, ARD, ZDF, SAT1 y PRO7 en Alemania; RTL, RAI1, RAI2, RAI3, Canale 5, Italia 1, Rete 4 en Italia; BBC1, BBC2, Channel 4, ITV, en el Reino Unido; La1, La2, TV3, Antena3 TV, Telecinco, Cuatro y La Sexta, en España, y France2, France3, M6 y TF1, en Francia. El análisis se limita a la programación de la franja de prime-time. La elección de esta franja responde a la voluntad de determinar el interés real de las cadenas por facilitar el consumo de documentales, situándolos de forma privilegiada en sus parrillas programáticas.

El universo de la investigación es el total de documentales y grandes reportajes programados en el prime-time de los países y cadenas apuntados más arriba; la muestra escogida son todos aquellos documentales aparecidos en la misma franja horaria de las mismas cadenas durante el periodo de una semana del mes de Enero del año 2011, concretamente la semana del 14 al 21 de Enero. La programación analizada se corresponde con la emitida en la franja horaria de prime-time de cada país, que varía en función de los hábitos televisivos y los estilos de vida. (Tabla 2).

\begin{tabular}{|l|c|}
\hline \multicolumn{2}{|c|}{ Tabla 2. Prime-time por países } \\
\hline España & $20: 30-22: 30$ \\
\hline Francia & $20: 00-22: 30$ \\
Italia & $20: 30-22: 30$ \\
Alemania & $19: 00-22: 30$ \\
Reino Unido & $20: 00-22: 30$ \\
\hline
\end{tabular}

Fuente: Euromonitor en Prado y Delgado, 2010:55

La elección de los países responde a criterios de notoriedad, en tanto que son considerados como los cinco mayores mercados televisivos de Europa (Prado y Delgado, 2010: 53); la de las cadenas, a su asimilación a los siguientes parámetros: son televisiones generalistas y de ámbito nacional. La contemplación de canales de titularidad dispar no es un elemento disgregador de la muestra sino que se establece como una variable de análisis que aporta información de valor a la hora de caracterizar el panorama. En el caso concreto de las televisiones públicas, además, se introduce el tipo de financiación como variable adicional ya que uno de los objetivos planteados es delimitar en qué medida ésta puede afectar a la programación de documentales.

2 "Euromonitor es un observatorio permanente de la televisión en Europa, operativo desde 1989, fundado con apoyo del servicio de investigación de la radiotelevisión pública italiana (RAI) por un grupo de investigadores europeos: Paolo Baldi, Ian Connell, Emili Prado y Claus-Dieter Rath”. (Prado y Delgado, 2010:53) 
Por lo que a la división temática de los contenidos documentales analizados se refiere, esta investigación toma como modelo la delimitación de Bienvenido León (2009: 28) que establece las categorías siguientes: a) científicos; b) culturales; c) sociales; d) políticos e ideológicos. Aún así, se ha querido afinar un poco más la clasificación, por lo que se incluyen también dos subdivisiones de lo que León llama genéricamente "documentales científicos", siguiendo el criterio apuntado por Annette Hill (Hill, 2007: 47). Los documentales históricos y los de naturaleza se distinguen del resto de "documentales científicos", resultando así las siguientes categorías:

1. Historia: exponen y explican acontecimientos históricos y su principal función es dar a conocer unos hechos, unos personajes o unas situaciones que acaecieron en un pasado remoto.

2. Ciencia: tratan sobre asuntos relacionados directamente con alguna disciplina científica como la química, la astronomía, etc.

3. Naturaleza: exploran las características biológicas y las formas de vida de animales y plantas, presentan paisajes naturales desconocidos, etc.

4. Política: tratan sobre asuntos de interés público o sobre conflictos políticos, económicos o ideológicos.

5. Sociedad: presentan situaciones problemáticas de grupos sociales concretos, denuncian estados de opresión o injusticia, etc.

6. Cultura: hablan de acontecimientos culturales, personajes famosos del mundo de las artes y las humanidades, etc.

\section{La presencia de documentales en el prime-time europeo}

El análisis de la programación de las cadenas de la muestra confirma que la presencia tanto de documentales como de grandes reportajes en el prime-time de las televisiones europeas es irrelevante. Del total de minutos de la franja los dos géneros ocupan un tiempo acumulado que equivale al $4 \%$, del cual un $3 \%$ se refiere al documental y un $1 \%$ al gran reportaje. Asimismo, se corrobora que las cadenas públicas son las que muestran un mayor compromiso con estos dos géneros, ya que el $80 \%$ de los documentales y grandes reportajes ha aparecido en canales públicos frente al $20 \%$ restante, que lo ha hecho en operadores privados. En este apartado se muestran los resultados del análisis con mayor profundidad y detalle, en dos etapas: en la primera se exponen los resultados de cada país, caracterizando su panorama concreto; en la segunda, se realiza un estudio comparativo dibujando el panorama europeo, tomando como muestra los cinco mercados televisivos más importantes. Los resultados presentados son en base al número de minutos de programación que representa cada una de las categorías analizadas respecto al total de minutos del prime-time.

\subsection{Resultados por país (2010-2011)}

\subsubsection{Alemania}

El documental es un contenido residual en el prime-time de Alemania. En la semana analizada se han identificado un total de tres documentales o grandes reportajes en la franja programática de máxima audiencia, lo que denota una apuesta muy escasa por el género. En tiempo acumulado, el documental supone una proporción exigua, un $2 \%$ del total de la programación de prime-time. Los resultados contemplan también 
un docudrama, un producto híbrido que combina el documental y la ficción para contar un acontecimiento histórico. Pese a que la mayoría del contenido documental aparece ficcionado hay también una parte significativa de imágenes recurso y entrevistas, lo que lo sitúa entre el documental y el docudrama.

No son resultados que sorprendan especialmente, si se tienen en cuenta los datos proporcionados por estudios anteriores, que coronaban Alemania como líder en la apuesta por la ficción en horario de máxima audiencia. En la temporada 2008-2009 el documental suponía el $0,7 \%$ de la programación de prime-time en Alemania, hecho que contrastaba con el 23\% dedicado a la ficción seriada. (Prado y Delgado, 2010: $57)^{3}$. Los datos actuales dibujan una clara tendencia al mantenimiento de una presencia baja del documental y el gran reportaje en horario de prime-time.

Por lo que a las temáticas se refiere, los documentales programados pertenecen a las categorías de "Naturaleza" e "Historia". Uno de ellos es un episodio de la serie documental "LIFE", producida por la BBC; los otros dos documentales son obras únicas, una de ellas de producción británica ("Edward VIII und die Nazis") y la otra de producción nacional ("Luxus auf dem Meer").

\subsubsection{España}

Los resultados de España, en cambio, apuntan un acierta tendencia al alza. El artículo "La televisión generalista en la era digital: tendencias internacionales de programación" (Prado y Delgado, 2010) establecía que, en la temporada 2008-2009 el documental ocupaba un 1,6\% de la programación de prime-time. La investigación actual refleja un aumento en el número de documentales y grandes reportajes programados, ya que estos ocupan el $6 \%$ del tiempo total de esta franja horaria. En número de programas, de los 121 aparecidos en las cadenas españolas, 6 son documentales o grandes reportajes, hecho que, pese al aumento, representa una proporción baja.

Igualmente pobre es la distribución de los documentales por canales ya que se concentran todos en la segunda cadena de Televisión Española, La2. Se produce un fenómeno significativo de concentración del género únicamente en la televisión pública, que programa documentales (y no grandes reportajes) en prime-time pero siempre relegados a su segunda cadena, no generalista y de carácter básicamente cultural. La interpretación positiva de este dato es el hecho de que La2 (a diferencia de otros canales públicos) programa una ratio muy elevada de documentales que equivale prácticamente a uno por día; la negativa, que no se programan documentales ni siquiera en la primera cadena de la televisión pública.

Por lo que a la caracterización de los documentales programados se refiere hacemos alusión a tres parámetros: la temática, el formato y la producción. En la temporada 2000-2001 (según los datos expuestos en 2003 por Miquel Francés en La producción de documentales en la era digital) quedaba clara la predilección por los documentales de naturaleza, una tendencia que se mantiene aquí, siendo 3 de los 6 do-

3 Este estudio contempla una muestra de 4 semanas de la temporada 2008-2009, a diferencia de la muestra de una semana de esta investigación. No obstante, en tanto que hablamos en ambos casos de porcentajes en base a minutos de programación, se considera oportuna la comparación. 
cumentales analizados de este ámbito. La segunda temática más popular en cuanto a volumen de programación es la historia, con dos documentales programados. Estos dos documentales históricos pertenecen a la serie francesa "Apocalipsis, la Segunda Guerra Mundial", de 2009. El último documental es un programa cultural biográfico sobre la vida y obra del literato español Gonzalo Torrente Ballester.

Por lo que a la producción se refiere cabe destacar que solamente uno de los 6 documentales programados es nacional, mientras que los otros cinco son, o bien de producción francesa o bien británicos. En cuanto al formato también en todos los casos excepto en uno se trata de capítulos de series documentales más extensas o one-off y sólo el documental biográfico español puede clasificarse de "obra única".

Como información adicional destacamos también que La2 programa documentales en prime-time de lunes a viernes, llenando la franja del fin de semana con un programa de ficción y numerosos reportajes. En este sentido es importante destacar la apuesta de la segunda cadena por la información en profundidad ya que, si se tienen en consideración los current affairs, reportajes y documentales de forma acumulada, estos representan casi un $60 \%$ del total de horas de programación en prime-time. "Redes", "Tres14" o "Reportero de la historia" son algunos de los espacios fijos de reportaje que encontramos los fines de semana y que ponen de manifiesto esta predilección por el género informativo. El otro $40 \%$ corresponde fundamentalmente a la ficción.

\subsubsection{Francia}

La situación en Francia es aún más desesperanzada con respecto al documental. El análisis de la programación de prime-time revela que no hay ningún programa de este género, mientras que sí que está presente el gran reportaje, que representa un 3\% del total de la programación en minutos. Estos datos rompen por completo la tendencia apuntada en el estudio de la temporada 2008-2009 donde Francia encabezaba la apuesta por la información en prime-time (por detrás de España) de la cual el documental representaba un 15\%. (Prado y Delgado, 2010: 55)

El espacio programado es, no obstante, un programa con auténtica vocación documental y que merece ser caracterizado. Se trata del programa "Thalassa", presentado en Francia por Georges Fernoud, y programado en la tercera cadena estatal, France3. No es, en realidad, un único documental sino que se trata de un contenedor de grandes reportajes realizados en diversas partes del mundo. El episodio analizado tiene una duración de 110 minutos y consta de 4 grandes reportajes firmados por reporteros, directores y productores diferentes, todos nacionales. El tema común de todos los reportajes o grandes reportajes de la marca "Thalassa" es el mar, que puede expresarse de diferentes modos, configurando programas de temáticas diferentes: naturaleza, historia, sociedad, política, etc.

\subsubsection{Italia}

El caso de Italia es idéntico (o, si cabe, peor) al de Francia. El cotejo del horario de máxima audiencia revela un único programa documental (un 1\% de la programación total), en una parrilla dominada por la ficción. En tiempo de programación, esto supone que de los 5.040 minutos que componen el prime-time de todas las cadenas analizadas, el documental ocupa sólo 60. Esta era ya la tendencia observada por Matilde 
Delgado y Emili Prado en su estudio de la programación de la temporada 2008-2009, en el que identificaron que el documental representaba un 1,4\% del prime-time. (Prado y Delgado, 2010: 57)

El programa "Nati Liberi" es un espacio regular sobre naturaleza, dedicado en este caso al Parque Nacional de Nairobi. De positivo hay que destacar que se trata de una buena producción nacional, de amplios recursos e impresionantes paisajes; de negativo, por supuesto, la exigua atención a los documentales y los grandes reportajes que, a diferencia de otros países, tampoco va acompañada de una prolífica programación de reportajes u otros géneros de información. La escasez de documentales es un síntoma del abandono generalizado de la información en el prime-time ya que ésta tampoco se manifiesta en la forma de otros géneros, aparte de los noticiarios.

\subsubsection{Reino Unido}

Para todo lo expuesto hasta ahora existe una excepción, el Reino Unido: productor, exportador y ávido programador de documentales, incluso en horario prime-time. El género documental representa un $12 \%$ del total de su programación en horario de máxima audiencia, con presencia tanto en las cadenas públicas como en las privadas. Para la temporada 2008-2009 Información e Infoshow representaban el 57\% de la programación total de la franja (Prado y Delgado, 2010: 55), lo que evidenciaba ya una determinada predilección por los contenidos de realidad. En el decurso de la semana planteada se programaron un total de 10 documentales y grandes reportajes, distribuidos entre las 4 cadenas analizadas. La presencia de cada uno de los géneros se reparte en un $6 \%$ de documentales y el mismo porcentaje de grandes reportajes.

Esto confirma la tendencia apuntada por estudios anteriores: en la temporada 20082009 el documental representaba el 13,7\% del horario de máxima audiencia. Como información adicional (aunque las variables son diferentes y no es posible establecer comparaciones) introducimos aquí el estudio realizado por Bienvenido León sobre la temporada 2003-2004. Este análisis, que tenía como muestra los 14 países de la Unión Europea, establecía que el documental suponía el 5,5\% del total de la programación en la televisión pública y el 7,3\% de la privada, en el Reino Unido. (León, 2007: 95). Son cifras extremadamente elevadas si se comparan con las de Alemania, España, Francia e Italia y, sumadas al $12 \%$ de la temporada 2010-2011, dibujan un panorama saludable para los géneros estudiados.

Asimismo, la diversidad en la distribución por cadenas denota que no se trata de una apuesta "personal" de un canal sino que hace pensar en una determinada tradición nacional, en una predilección de los programadores británicos por el documental y el gran reportaje. Otro elemento muy destacable es la variedad temática de los contenidos programados en esta materia. Todas las categorías de la taxonomía apuntada en la metodología aparecen en mayor o menor grado en la muestra, por lo que existe una gran diversidad de temas, la presencia de los cuales, además, es equilibrada.

\subsection{Resultados comparativos (UE-5 2010-2011)}

La revisión individual de la situación del documental y el gran reportaje en los cinco grandes mercados de la Unión Europea (UE-5) hace evidente una primera conclusión: la presencia de estos géneros en prime-time es dispar en los cinco mercados y, en ge- 
neral, escasa. Sólo el Reino Unido hace una apuesta firme y generalizada por los espacios documentales, expresada en la variedad de temas tratados y la dispersión de los géneros en los diversos canales, tanto públicos como privados.

El documental y el gran reportaje representan aproximadamente un $2,4 \%$ de la programación de prime-time de los cuatro mercados continentales, una media que asciende al 4\% para la UE-5 al incluir el porcentaje británico. Así pues, incluso con los datos acumulados de los cinco mercados el resultado final es irrisorio.

Una posible explicación para el reducido exponente del género podría ser la necesidad de balancear los programas entre un número muy abundante de géneros con el fin de obtener una programación variada en contenidos. Pero estudios anteriores demuestran que, nada más lejos de la realidad, algunos géneros de ficción generan porcentajes de programación desorbitados. En relación con la temporada 2008-2009, por ejemplo, las series de televisión suponían el $22 \%$ de los contenidos para Francia, el $23 \%$ para Alemania y el $21 \%$ para Italia, donde los documentales eran el $4,8 \%$, el 0,6\% y el 1,4\%, respectivamente. (Prado y Delgado, 2010: 57).

GRÁFICO1: Presencia de documentales en el prime-time UE-5 Temporada 2010-2011 (en minutos)

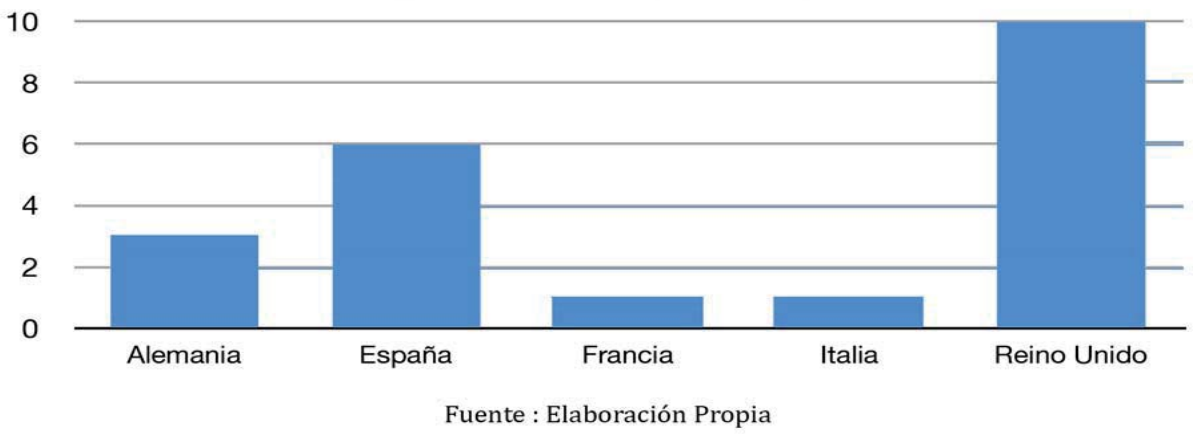

Tampoco ofrece sorpresas la comparación entre cadenas públicas y operadores privados. E1 80\% de los documentales y grandes reportajes han aparecido en televisiones públicas, frente al $20 \%$ programado en operadores privados. Los casos más relevantes en este sentido son los de España y el Reino Unido. El primero es importante por tener todos los programas del género en la misma cadena pública (con 6 documentales, es el segundo país que más apuesta por ellos pero únicamente en la segunda cadena de la televisión estatal); el segundo es el Reino Unido, por ser el único mercado que cuenta con documentales en las cadenas privadas, tanto en Channel 4 como en ITV.

Estos datos coinciden en corroborar tendencias más generales apuntadas por estudios basados en temporadas anteriores. En su artículo "Commercialization and Programming Strategies of European Public Television" (2007), Bienvenido León aporta que, en porcentaje de espacios, las televisiones públicas programaron un $8.1 \%$ de documentales, mientras que las privadas lo hicieron sólo en un 2.3\%. (León, 2007: 93). En general, la información ostentó el 38\% de la programación de las cadenas públicas mientras que supuso sólo el 27\% para las privadas. (León, 2007: 90). En 2008- 
2009, la información representaba el 40,9\% para las públicas y el 22,22\% para las privadas. (Prado y Delgado, 2010: 58).

En cuanto a las diferencias entre cadenas públicas en función del origen de sus ingresos, los resultados apuntan a la existencia de una relación directa entre el tipo de financiación y la programación de documentales. De un total de 17 documentales o grandes reportajes aparecidos en las televisiones públicas, 12 es el número acumulado que representan la televisión pública británica (la BBC1 i la BBC2) i la española (La2), las únicas financiadas íntegramente a partir de fondos públicos (ya sean presupuestos o cánones) y sin publicidad. Es así como los dos países con apuestas firmes por el género son también aquellos en los que la televisión pública funciona sin ataduras publicitarias. De forma contraria, en Alemania, Francia e Italia, donde los fondos públicos se combinan con ingresos procedentes de espacios publicitarios, el documental es un contenido altamente residual debido, seguramente, a su escasa rentabilidad comercial.

Pese a ello, Bienvenido León desmiente esta relación directa en su muestra de 2003-2004. Su estudio de 2007 establece que "surprisingly, the level of entertainment is not necessarily higher in those public channels which have a greater deal on dependence on advertising". (León, 2007: 90). Los resultados de su comparación llevan a León a interpretarlos como una evidencia de que el mercado no es el único factor que influencia la programación sino que existen otros condicionantes socio-políticos que influyen en la selección de contenidos (León, 2007: 91). En cualquier caso, nuestra observación actual confirma que las televisiones públicas de financiación mixta programan menos documentales que las televisiones públicas de financiación estatal.

Quizás el documental sea un caso extremo de impopularidad y sea por esto que las diferencias entre unas y otras televisiones públicas se hagan aquí más patentes y hagan intuir que, con respecto a estos géneros, el mercado sí que tiene la última palabra en términos de emisión en el prime-time. Lo apuntaba ya Zoellner en su estudio de 2009 al afirmar que "reality- and celebrity-based factual entertainment programs, that promise high ratings and are relatively cheap to reproduce, are therefore on the rise, whereas documentary producers criticise sinking or stagnating budgets, staff cuts, and a reduction of broadcasting strands for one-off, international, and auteur documentaries that contribute to the increased economic pressure and competition amongst independent documentary producers" (Zoellner, 2009: 509). Los imperativos comerciales son los que apartan el documental de la franja prime-time en las cadenas europeas, tanto por su inexistencia en las televisiones privadas como por su escasez en aquellas televisiones públicas con responsabilidades con sus clientes publicitarios.

Otro criterio de comparación que se ha utilizado en este caso es el que concierne al origen de los documentales programados, ya que la producción doméstica de los mismos podría considerarse otro indicativo del grado de interés de cada país por el género. Como era de esperar, el mayor productor de documentales es también el Reino Unido, que consigue un pleno en la relación entre el número de documentales programados y la producción doméstica. También el resto de mercados han emitido algún documental de producción nacional, pero tanto Alemania como España programan mas documentales extranjeros que domésticos. De los seis aparecidos en La2, cinco 
eran foráneos y sólo uno era español; dos de los aparecidos en las pantallas alemanas eran británicos y sólo uno era de producción nacional. A la luz de los resultados, el Reino Unido es el principal exportador de documentales con dos programas emitidos en Alemania y tres en España. De estos cinco, cuatro llevaban la marca BBC. Italia y Francia, pese a haber programado un sólo documental en el prime-time exponen productos nacionales de los cuales destacan unos recursos de producción considerables.

Son resultados que contrastan con los expuestos por Miquel Francés en base a los datos obtenidos de GECA (2001). Francés apuntaba el "alto nivel de producción nacional de documentales que emite La2" (Francés, 2003: 150) que estimaba en un 90\% y que contrasta con los datos presentados. Nuestro análisis demuestra que esta proporción no se cumple actualmente, por lo menos en horario de prime-time. Quizás se estén dando cambios en el ámbito de la producción o quizás sea tan sólo una cuestión de franjas horarias pero lo cierto es que los resultados obtenidos contrastan también con los de otras temporadas: en 1998 había 5.390 documentales de producción española en televisión por cada 2.468 de producción europea; un año más tarde, los documentales nacionales superaban también con creces a los comprados a otros mercados (Francés, 2003: 81). La política actual de compra de programas documentales por parte de algunas televisiones europeas muestra también una predilección por el formato de documental seriado. Los datos obtenidos ponen de manifiesto esta tendencia ya que de los 22 documentales programados 13 son capítulos de una serie más amplia, el número de episodios de la cual varía en cada caso.

El ejemplo más claro de esta tendencia a la compra de series extranjeras es el de España donde cinco de los 6 documentales son capítulos de dos series: "Apocalipsis: La Segunda Guerra Mundial", de producción francesa y "Planeta Tierra", de producción Inglesa. Son ejemplos de lo que Francés llama "documentales televisivos seriados de divulgación", normalmente producidos por empresas independientes clásicas y consolidadas (como en el primer caso) o por grandes cadenas de televisión con tradición de producción documental (como en el segundo caso). Suelen ser documentales estrenados en prime-time en el país de origen "para pasar después a la multidifusión y a la distribución en DVD home". (Francés, 2003: 84). Éste es también el caso de las series "Life" (emitida en la ARD), "Human Planet" o "Natural World", estrenadas en la BBC. El coste de producción de cada capítulo de estas series se estima alrededor de 260.000 euros (Francés, 2003: 85) por lo que sólo las grandes cadenas con extensos presupuestos destinados al documental pueden permitírselo. La tendencia de los países donde el presupuesto es mucho más bajo (como España) es a comprar este tipo de contenidos.

Un último factor a considerar en el dibujo del panorama documental en el primetime de la televisión europea es el criterio temático de selección en los programas. Hasta aquí se han apuntado dos tendencias básicas: el predominio de los programas de naturaleza e historia y la poca variedad temática en los documentales programados por los países europeos, exceptuando el Reino Unido. El gráfico comparativo confirma las dos inclinaciones: la presencia de los documentales de naturaleza en los cinco países corroboran el liderazgo de esta temática que, además, es mayoritaria en todos los mercados, excepto en Alemania. Le sigue de cerca la historia, con importante presencia en tres de los cinco y los documentales sociales y los culturales, siendo la ciencia y la política los ámbitos menos abordados. En la distribución por países el 
Reino Unido destaca ostensiblemente, por la variedad temática de sus programas documentales, que representan todas las categorías determinadas en la metodología.

GRÁFICO2: Distribución de los documentales por temáticas en el prime-time UE-5 Temporada 2010-2011 (número)

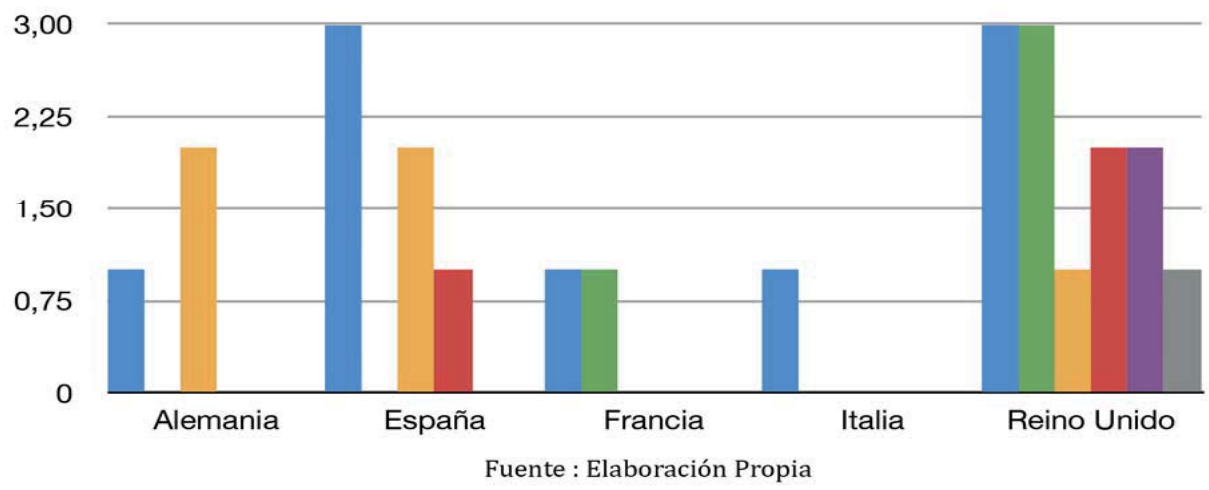

\section{Conclusiones}

La televisión europea (UE-5) actual apuesta poco por el documental y el gran reportaje. Su presencia en el prime-time es simbólica (apenas un $4 \%$ del total de la programación) y se sitúa fundamentalmente en las cadenas públicas, a menudo en los segundos canales. La mayoría de los mercados, además, practican una política de compra más que de producción en el área documental, hecho que sugiere la atribución de los recursos de producción a otros géneros de mayor rentabilidad comercial. La falta de rentabilidad como causa de la escasa presencia de los espacios documentales en pantalla se deduce de dos constataciones: la ausencia generalizada en las televisiones comerciales y la exigüidad en los canales públicos que cuentan con sistemas mixtos de financiación.

Las televisiones, incluso las públicas, evitan el riesgo a toda costa; apuestan por la ficción como caballo ganador y marginan todo lo susceptible de producir índices de audiencia bajos (aunque pueda producir también un placer estético, un conocimiento de valor o una determinada visión sobre el mundo y sus problemas). La lógica de mercado se ha impuesto sobre la de servicio, en un panorama marcado por la competencia agresiva entre todos los agentes. "Television schedules are heavily formatted, which involves predetermined standards for form and content elements that aim to meet audience expectations and increase audience loyalty through familiarity and predictability". (Zoellner, 2010: 521)

La desaparición del documental de las pantallas de la televisión pública es un síntoma importante de esta tendencia, tanto por el tradicional arraigo entre género y medio como por la aparente idoneidad del primero como contenido de la televisión pública; una televisión pública que se aleja cada vez más de la noción de servicio. "Hasta hace poco más de una década, esta noción significaba dar un servicio televisivo de calidad, que pudiera contribuir a mejorar el nivel cultural de la población. Hoy parece que servicio público significa satisfacer a la mayor cantidad de público. Con una ló- 
gica simplista se nos quiere convencer de que un servicio público televisivo cumple mejor su función cuanto más público lo ve.” (Aznar, 2002: web).

Es así como la popularidad se ha convertido en el criterio básico (y único en el las televisiones privadas) para el diseño de las parrillas programáticas. Un estudio realizado en 2001 de los 20 programas más populares del año para las audiencias de todos los países de la Unión Europea establecía que el 32\% pertenecían al género deportes; el $25 \%$ a series de ficción, el 7,5\% al macrogénero información y el 5,1\% restante eran programas musicales (La Porte Alfaro y Sádaba, 2001: 7). Son datos que nos hablan de las preferencias del público y que dan una idea del porqué de las tendencias apuntadas en la investigación.

Hugo Aznar reflexiona sobre estas cuestiones en su artículo "Televisión, telebasura y audiencia: condiciones para la elección libre" (2002) donde plantea la necesidad de revisar los criterios de selección de los programas, especialmente en la televisión pública. Su objetivo es desbancar el criterio de "popularidad" como único patrón así como cuestionar los métodos de medida de esta popularidad. Para Aznar "un requisito habitual para que una elección pueda considerarse verdaderamente libre es que haya varias alternativas. Y no cualesquiera alternativas: entre ellas debe existir un cierto grado de variedad (para que sean realmente diferentes) y de comparabilidad (para poderlas contrastar entre sí)." (Aznar, 2002: web). Esto implica, sin duda, la asunción de cierto grado de riesgo a la hora de programar, prevaliendo criterios de educación y transmisión cultural por encima de los índices de audiencia y apostando por formatos, temáticas y géneros diversos.

Según este punto de vista resulta complicado afirmar que el documental o el gran reportaje sean géneros impopulares ya que parten de una marginalidad que afecta no sólo al número acumulado de programas aparecidos sino también al horario en el que se programan y al tipo de canales. Lo mismo sucede con tantos otros géneros que, como éste, no suelen ser una opción clara para la audiencia y, en consecuencia, obtienen índices de popularidad muy pobres. Pero incluso aceptando que sean géneros impopulares ¿podemos permitir que sea éste un criterio dogmático para la selección de contenidos de las televisiones públicas?

"La gente que defiende el reino de los índices de audiencia pretende que no hay nada más democrático, que hay que dejar a la gente la libertad de juzgar, de elegir." (Bourdieu, 1997: 96). Pero la televisión pública tiene obligaciones, más allá de dar a la gente "lo que quiere"; tiene también la función de transmitir conocimientos y proyectar el patrimonio cultural, (Francés, 2003: 107) y su cometido debe reflejarse en "la programación de contenidos de calidad, la independencia política y la no subordinación a los intereses comerciales" (Manfredi Sánchez, 2008: 12).

Con respecto al documental, la investigación planteada hasta aquí determina que su presencia no se equipara a otros géneros más populares, como el cine o las series de ficción. Establece, además, que la causa de su ausencia enraíza con su escasa rentabilidad comercial tanto en las televisiones privadas como en las públicas, donde la disparidad de los géneros es muy acusada en horario de prime-time. Advierte, así, que se trata de una realidad contradictoria con los principios de servicio público establecidos por el Consejo de Europa, (The Council of Europe, 2004: 1) que requiere variedad, educación, cultura e información a la televisión pública, considerando que el 
documental es un género idóneo para la consecución de estos objetivos. Las soluciones con respecto a la popularidad del documental no pasan por eliminarlo de las parrillas o por relegarlo a segundos canales. La experiencia del Reino Unido demuestra que otra realidad es posible, una en la que los documentales gocen de buenos índices de audiencia y en la que una televisión pública con un $12 \%$ de documentales en el horario de máxima audiencia sea la más popular.

Quizás las políticas de producción documental deban renovarse y buscar la manera de hacer productos atractivos sin traicionar sus fundamentos o quizás sea necesario simplemente incrementar su presencia en pantalla, con el fin de conseguir resultados positivos. Anna Zoellner propone que "In an attempt to increase its audience appeal broadcasters could similarly invest into the production value and innovation potential of documentary with the aim to create more challenging and enlightening accounts of reality that will contribute to social knowledge and discourse". (Zoellner, 2009: 534). Para Zoellner, la popularidad depende de si las televisiones públicas apuestan por el documental, invirtiendo y revisitando el género, o dejan que caiga en el olvido, alegando su escasa audiencia. En este sentido, la BBC (pero también el resto de canales británicos) dibujan un camino a seguir, si lo que se persigue es una televisión pública con diversidad de géneros y temas, entre los que el documental y el gran reportaje puedan jugar un papel fundamental.

\section{Referencias bibliográficas}

AZNAR, Hugo (2002): "Televisión, telebasura y audiencia: condiciones para la elección libre", en Revista Latina de Comunicación Social, 48: http://www.ull.es/publicaciones/latina/2002/latina48marzo/4807aznar2.htm [Fecha de consulta: 3 de Septiembre de 2013]

BERELSON, Bernard (1952): Content analysis in communication research. New York, Hafner Publishing Company.

BOURDIEU, Pierre (1997): Sobre la televisión. Barcelona, Anagrama.

BRUNDSON, Charlotte; JOHNSOSN, Catherine; MOSELEY, Rachel; WHEATLEY, Helen (2001): "Factual entertainment on British television: The Midlands TV Research Group's 8-9 Project". European Journal of Cultural Studies, vol. 4 nº 1 , pp.29-62.

BUSTAMANTE, Enrique (1999): La televisión económica: Financiación, estrategias y mercados. Barcelona, Gedisa.

CHAPMAN, Jane; ALLISON, Kate (2009): Issues in contemporary documentary. Cambridge, UK; Malden, MA, Polity.

COLES, Gail (2000): "Docusoap: Actuality and the Serial Format", en CARSONAND, Bruce y LLEWELLYN-JONES, Margaret: Frames and Fictions on Television: The Politics of Identity within Drama. Exeter, Intellect Books, pp. 27-39.

CORNER, John (2000): "What can we say about documentary?". Media Culture \& Society, n²2 (5), pp. 681-688. 
COUNCIL OF EUROPE (2004): Public Service Broadcasting: Report of the Committee on Culture, Science and Education, Doc. 10029; at: http://assembly.coe.int /Documents/WorkingDocs/doc04/EDOC10029.htm [Fecha de consulta: 10 de Septiembre de 2010].

FRANCÉS, Miquel (2003): La producción de documentales en la era digital: Modalidades, historia y multidifusión. Madrid, Cátedra.

HILL, Annette (2007): Restyling factual TV :Audiences and news, documentary and reality genres. London and New York, Routledge.

KILBORN, Richard (1998): "Shaping the Real: Democratization and Commodification in UK Factual Broadcasting." European Journal of Communication, vol.13, pp. 201-218.

KILBORN, Richard (2000): From Grierson to the docu-soap: breaking the boundaries. Luton, University of Luton, cop.

LA PORTE ALFARO, Teresa; SÁDABA, Teresa; (2001): "Globalisation of the media industry and possible threats to cultural diversity". European Parliament, Directorate General for Research, Directorate A, The STOA Programme.

LEÓN, Bienvenido (2007): "Commercialisation and Programming Strategies of European Public Television. A Comparative Study of Purpose, Genres and Diversity”. Observatorio (OBS*) Journal n², pp.81-102.

LEÓN, Bienvenido (2009): Dirección de documentales para televisión: Guión, producción y realización. Pamplona, Eunsa.

MANFREDI SÁNCHEZ, Juan Luis (2008): La televisión pública en Europa. Madrid, Fundación Autor.

PRADO, Emili (1995): "Programación y televisiones públicas" en: La nueva perspectiva audiovisual. El reto de la televisión pública regional. Madrid, Telemadrid.

PRADO, Emili (2002): “Telerrealidad: globalización y uniformización” en: VIDAL BENEYTO, José: La ventana global. Madrid, Taurus.

PRADO, Emili y DELGADO, Matilde (2010): "La televisión generalista en la era digital. Tendencias internacionales de programación". Telos, $\mathrm{n}^{\circ}$ 84. Madrid, Fundación Telefónica.

RICHERI, Giuseppe (1994): La transición de la televisión: Análisis del audiovisual como empresa de comunicación. Barcelona, Bosch.

ZOELLNER, Anna (2009): "Professional Ideology and Program Conventions: Documentary Development in Independent British Television Production" Mass Comunication and Society, vol. $12 \mathrm{n}^{\circ}$ 4, pp. 505-536. 